\title{
Grounding is not a strict order ${ }^{1}$
}

\author{
Gonzalo Rodriguez-Pereyra \\ University of Oxford
}

1. A strict order is a relation that is transitive, irreflexive, and asymmetric. My aim in this paper is to argue that grounding is not a strict order. More precisely, I shall argue that grounding is neither transitive, nor irreflexive, nor asymmetric. ${ }^{2}$ This is controversial and original. It is controversial because some philosophers maintain that grounding is a strict order, or that it has some of the three features necessary for being a strict order (for instance, Correia (2010) and Raven (2013: 193-94) think it has all three; Fine thinks it is irreflexive and transitive (2010: 100), which entails it is also asymmetric (see also Fine 2012: 56); Audi, Bennett, Rosen, and Schaffer think that it is irreflexive and asymmetric (Audi 2012a: 102, Audi 2012b: 691-92, Bennett forthcoming, Schaffer 2009: 364, Rosen 2010: 115-116)). And it is original because although some philosophers have maintained that grounding is not a strict order because of lacking one of the three features, as far as I know no one has argued that it lacks all three of them (Schaffer (2012) has argued that grounding is not transitive, Thompson (2014) has argued that it is not asymmetric, and Correia (2014) has rejected its irreflexivity). ${ }^{3}$ Furthermore the way in which I shall argue for my thesis is original, since some of my examples that grounding is neither irreflexive, nor asymmetric, nor transitive will be examples of truthmaking where irreflexivity, asymmetry, and transitivity fail.

The structure of the paper is as follows. In Section 2 I shall characterize grounding in a general way. From this characterization it will follow that

\footnotetext{
${ }^{1}$ I thank Paul Audi, Anita Avramides, Eduardo Barrio, Tomás Castagnino, Michael Clark, Kit Fine, Bob Frazier, Dan Giberman, Ghislain Guigon, Dan Isaacson, Nick Jones, David Liggins, Dan Lopez de Sá, Penelope Mackie, Anna-Sofia Maurin, Michael Peramatzis, Federico Pailos, Josh Parsons, Bryan Pickel, Martin Pickup, Lavinia Picollo, Howard Robinson, Luis Robledo, Sven Rosenkrantz, Jonathan Schaffer, Christopher Shields, Damian Szmuc, Tuomas Tahko, Diego Tajer, Paula Teijeiro, Alastair Wilson, Elia Zardini, Ezequiel Zerbudis, three referees, and audiences in Barcelona, Buenos Aires, Manchester, and Oxford for comments and discussion.

${ }^{2}$ A referee has suggested that I should use the phrase 'strict partial order', but in fact I am not going to question the partial character of grounding, i.e. the fact that grounding is not connected. Thus I shall stick with 'strict order'.

${ }^{3}$ Jenkins (2011) has argued that one should not assume that grounding is irreflexive, and Barnes (2014) has argued that dependence is not asymmetric, but she distinguishes dependence from grounding.
} 
truthmaking and another relation that I shall call alethic-fact grounding are species of grounding. Thus if either of these relations lacks one of the features necessary for being a strict order, neither is grounding a strict order. In section 3 I shall discuss and reject Schaffer's counterexamples to the transitivity of grounding. In section $4 \mathrm{I}$ shall present my case against the transitivity of truthmaking. In section 5 I shall present my case against the irreflexivity of truthmaking and alethic-fact grounding. In section 6 I shall present my case against the asymmetry of truthmaking and alethic-fact grounding. In section 7 I shall argue that a contrastive quaternary relation of grounding, like the one proposed by Schaffer (2012: 130) is still irreflexive and asymmetric. Section 8 is a brief conclusion.

2. Truthmaking is the relation that obtains between a true proposition and that in virtue of which it is true, its truthmaker. For instance, according to many, the fact that Socrates is white is the truthmaker of the proposition < Socrates is white $>{ }^{4}$ So the fact that Socrates is white and the proposition < Socrates is white> are related by the truthmaking relation. Similarly, according to many, Socrates is the truthmaker of the proposition $<$ Socrates exists $>$. So Socrates and the proposition $<$ Socrates exists $>$ are related by the truthmaking relation.

Is truthmaking a case of grounding? I think it is, and others think so as well (Schaffer 2009: 375, Liggins 2012: 269; cf. Schnieder 2006: 30). The relation between truthmaker and truth seems analogous to the relation between member and set, between part and whole, and between being a cruel action and being a wrong action, and these are all initially plausible cases of ground and grounded. It is thus initially plausible that truthmaking is a case of grounding.

Sometimes grounding is characterized as a non-causal relation of dependence (Audi 2012a: 101, 2012b: 690). But then truthmaking is not a case of grounding, since the proposition $<$ Socrates is white $>$ does not depend on the fact that Socrates is white. What depends on the fact that Socrates is white is not the proposition itself, but its truth or its having the property of being true. In

\footnotetext{
${ }^{4}$ As is nowadays usual, I am using $<\mathrm{P}>$ to stand for the proposition that $\mathrm{P}$, and $<$ Socrates is white $>$ to stand for the proposition that Socrates is white. And I shall let [P] stand for the fact that P.
} 
other words, it is not the fact that the proposition exists that depends on the fact that Socrates is white, but the fact that the proposition is true.

But, as I said, truthmaking seems to be a case of grounding. Thus the considerations of the previous paragraph suggest that grounding should not be characterized as a non-causal relation of dependence. And yet there seems to be something right in the idea of grounding as a non-causal relation of dependence.

I think the way out of the dilemma is the following. Grounding is the noncausal generic relation of being $F$ in virtue of (or, equivalently, the generic relation of being F non-causally in virtue of). Thus a relation is a case or species of grounding if it is a specification of the non-causal generic relation of being $F$ in virtue of. Therefore truthmaking is a case or species of grounding, since it is the non-causal relation of being true in virtue of: the proposition <Socrates is white> is true in virtue of the fact that Socrates is white. Similarly, being right in virtue of, being blue in virtue of, existing in virtue of, and many other such relations are also cases or species of grounding.

Thus truthmaking is a species of grounding. But Kit Fine has argued that truthmaking is not a case of grounding, even if they are close cousins. One thing he says is that grounding, if a relation, should hold between entities of the same type and, insofar as a choice needs to be made, such entities should probably be facts rather than proposition (2012: 43; Fine's own view is that grounding is an operation rather than a relation). This excludes truthmaking as a case of grounding since the truthmaking relation links entities of different kinds to propositions. But no reason has been given why ground should only link entities of the same type. And, indeed, other grounding theorists take ground to link entities of different kinds (Schaffer 2009: 375).

Fine objects to truthmaking in other ways too. For instance, he argues that truthmaking theory has an unduly restricted conception of what grounds and of what is grounded, and that it is ill-suited to the step-by-step procedure to determine what grounds what (2012: 43-44). But these are objections to the project of letting truthmaking theory play all the roles associated to a theory of grounding. They are not objections to the more limited claim, which is my claim here, that truthmaking is a species of grounding. Fine also objects that the relation of truthmaking is defined in modal terms, i.e. as the relation of 
necessitation, and that this has, among others, the problem that any necessary truth will be grounded by anything (2012: 45). But there is no need to define truthmaking as necessitation and, in fact, truthmaker theorists often define the truthmaking relation in terms of being true in virtue of, e.g. Armstrong 2004: 5, 7, 16-17, Rodriguez-Pereyra 2002: 34, 2005: 17-18. Thus, although Fine is right that there is more to grounding than truthmaking, nothing he has said seems to undermine the idea that truthmaking is a species of grounding.

Of course, some, Fine included, may reject my claim that truthmaking is a species of grounding by rejecting my general characterization of grounding as the non-causal generic relation of being $F$ in virtue of. They might think that grounding should be characterized as the relation of in virtue of, or as being F in virtue of being $G$, or in some other way. But given the scope and plan of the paper I cannot engage in defending my general characterization of grounding. It is initially plausible that truthmaking is a species of grounding and all I can do here is to give reasons why this is the case, but I cannot defend those reasons. For my aim is to argue that grounding is not a strict order, and I will do so by arguing that two species of grounding relations, one of which is truthmaking, fail to satisfy the definition of a strict order. Even those who think that truthmaking is not a species of grounding could still be persuaded by my argument that the other grounding relation is not a strict order and so that, in general, grounding is not a strict order (though I need the claim that truthmaking is a species of grounding to reach my conclusion that grounding is not transitive).

What is the other relation I shall be discussing here? It is a close relative of truthmaking. Think of a truthmaker and the proposition it makes true. Because of the truthmaker, there is a fact that the proposition is true. And so the fact that the proposition is true exists, or obtains, in virtue of the truthmaker. The fact that the proposition is true and the truthmaker stand in the non-causal exists (or obtains) in virtue of relation. Thus the truthmaker grounds the fact that the proposition is true. For instance, Socrates grounds the fact that $<$ Socrates exists $>$ is true, since the latter fact exists or obtains in virtue of Socrates. Similarly, the fact that Socrates is white grounds the fact that <Socrates is white $>$ is true, since the latter fact exists or obtains in virtue of the fact that Socrates is white. I shall call this relation, obtaining between a truthmaker and the fact that 
the proposition it truthmakes is true, the relation of alethic-fact grounding. (Note that taking these two relations as cases of grounding commits one to grounding not being a relation that obtains only between facts. This is controversial in the theory of grounding. There are those who maintain that grounding can obtain only between facts (e.g. Audi 2012a and Rosen 2010; cf. Fine 2012: 43) and those who maintain it can obtain between other entities too (e.g. Schaffer 2009)).

So both truthmaking and alethic-fact grounding are species of grounding. But for the genus to be a strict order is for all its species so to be. Thus by showing that neither truthmaking nor alethic-fact grounding are strict orders, I shall have shown that grounding is not a strict order either.

Whenever an entity is a truthmaker of a certain proposition, that entity is the alethic-fact ground of the fact that that proposition is true. The relations of truthmaking and alethic-fact grounding are thus closely linked. But this close link does not mean that they must have the same formal properties. So the fact that truthmaking is neither transitive, nor irreflexive, nor asymmetric, does not mean that alethic-fact grounding has none of those three formal properties. Thus my examples that truthmaking is neither irreflexive nor asymmetric and my examples that alethic-fact grounding is neither irreflexive nor asymmetric will be independent from each other - although they will be similar. Furthermore, although I shall argue that truthmaking is not transitive, I am not in a position to argue that alethic-fact grounding is not transitive. But I shall first argue that Schaffer has not established that grounding is not a strict order since he has not established that grounding is not transitive.

3. Schaffer has presented the following counterexample to transitivity. Consider the set $\mathrm{S}=\{a, b, c\}$. Schaffer argues that (a) the fact that $c$ is a member of $\mathrm{S}$ grounds the fact that $S$ has exactly three members and (b) the fact that $S$ has exactly three members grounds the fact that $S$ has finitely many members, but (c) it is not the case that the fact that $c$ is a member of $S$ grounds the fact that $S$ has finitely many members (Schaffer 2012: 127-128).

Schaffer supports (a) by saying that if $c$ were not a member of $S$ then $S$ would have had two members and not three. Given that sets have their members necessarily, it is controversial that if S had not had $c$ as a member, it would have 
had two members. But it is still plausible that if it had not had $c$ as a member, it would not have had three members, since it would not have existed. Still, Schaffer's reasoning treats the fact that something is counterfactually necessary for another fact as a sufficient condition of the former fact being a ground of the latter, where a fact [P] is counterfactually necessary for a fact [Q] if and only if had [P] not obtained, [Q] would not have obtained.

This differs from Schaffer's support for (c). For he says that (c) is true because $S$ would have been finite even without $c$ as a member. But, it might be objected, $\mathrm{S}$ would not have been finite without $c$ as a member, since $\mathrm{S}$ would not have existed. This problem can be circumvented by changing the example and using a property that contingently has three instances (as noted by Litland 2013: 25-26). So I shall ignore this problem and continue using Schaffer's example of set $\mathrm{S}$.

So, according to Schaffer, the fact that $c$ is a member of S does not ground the fact that $S$ has finitely many members because if $c$ had not been a member of $\mathrm{S}, \mathrm{S}$ would still have been finite. But this means that a fact being counterfactually necessary for another fact is a necessary condition of the former grounding the latter. And given what he says about (a), that a fact is counterfactually necessary for another fact is necessary and sufficient for the former grounding the latter.

But that a fact is counterfactually necessary for another fact is a necessary condition for the former grounding the latter conflicts with (b), since the fact that $S$ has exactly three members is not counterfactually necessary for the fact that S has finitely many members. But all Schaffer says about (b) is that it is plausible because having finitely many members is a determinable, of which having exactly three members is a determinate (2012: 127). So perhaps the idea is that, provided property $F$ is not a determinate of property $G$, if the fact that something is F grounds the fact that it is G, that it is F must be counterfactually necessary for its being G. But it is not clear what the rationale for exempting the case of determinates and determinables would be. Furthermore, it seems that other exceptions will be needed since it is a standard idea that disjunctive facts are grounded in each of their true disjuncts, despite the latter often not being counterfactually necessary for the former. But then what is difficult to see is why in the case of the facts that $c$ is a member of $S$ and that $S$ has finitely many 
members, for the former to ground the latter, the former must be counterfactually necessary for the latter.

Now, in private correspondence Schaffer has said that his intention was to use the counterfactual test not to establish his claims (a) and (c) but as a heuristic to buttress the intuitions that (a) and (c) are correct. But since no reason has been given for why the counterfactual test is a good heuristic in the cases in which it is supposed to be a good heuristic, it is not clear that it buttresses any intuitions. Furthermore, this methodology seems impotent to convince anyone who does not share the initial intuitions. This is my own case. Indeed, I reject (a). For the identity of its members has nothing to do with the fact that $S$ has exactly three of them - what grounds the fact that $S$ has exactly three members is the fact that there is something, something else, and a third thing such that none of them is identical with either of the others, all three are members of $S$, and nothing else is. ${ }^{5}$

Schaffer produces two other counterexamples to the transitivity of grounding. One is the dented sphere case. But this case has exactly the same structure as the case of the three-membered set $\mathrm{S}$, and so what I said about the latter applies to the former. The other case is that of Cadmus' meow. According to Schaffer, (d) the fact that the creature was produced from the meeting of this sperm and that ovum grounds the fact that Cadmus is meowing and (e) the fact that Cadmus is meowing grounds the fact that something is meowing, but (f) the fact that the creature was produced from the meeting of this sperm and that ovum does not ground the fact that something is meowing. Given what Schaffer says about (d), (e), and (f), it is not clear that this case is intended to have the same structure as the other two.

I think this example is not a failure of transitivity. In defense of (d) Schaffer says that, given origin essentialism, (d) is plausible, since being produced from the meeting of this sperm and that ovum helps make the creature Cadmus as opposed to some other cat. If one wonders why Cadmus is meowing,

\footnotetext{
${ }^{5}$ In private correspondence Schaffer has noted that what grounds the existential generalization that there is something that is a member of $S$ is the fact that $c$ is a member of $S$ and that, therefore, the believer in transitivity will nevertheless have to admit that the fact that $\mathrm{c}$ is a member of $S$ grounds the fact that $S$ has exactly three members. This is a nice point, and it means that the defender of the transitivity of grounding cannot reject (a) on the basis I have proposed. But since I do not accept the transitivity of grounding, I can reject (a) on that basis.
} 
Schaffer says, the facts that make the meowing creature Cadmus are part of the reason (2012: 128). So the grounded fact is the fact that Cadmus is meowing. This is not the fact that Cadmus is meowing, since this fact is not grounded by what makes the creature Cadmus - what makes the creature Cadmus has nothing to do with the fact that it is meowing.

And Schaffer says that (e) is plausible since the fact that Cadmus is meowing provides a factual witness for the existential generalization that something is meowing, and factual witnesses ground existential generalizations. If one wonders why something is meowing, Schaffer says, the fact that Cadmus is meowing provides a sufficient reason (2012: 128).

Note that given his commitments in the case of set S, Schaffer will have some explaining to do concerning why (e) is acceptable, since the fact that Cadmus is meowing is not counterfactually necessary for the fact that something is meowing. But, independently of this, what grounds the fact that something is meowing is not that Cadmus is meowing but that Cadmus is meowing. That Cadmus is meowing grounds the fact that Cadmus is doing something, not that something is meowing. That in virtue of which something is meowing is that Cadmus is meowing, not that Cadmus is meowing. And so there is no failure of transitivity in this case since the grounded in (d) is not the grounding in (e).

This presupposes that the facts that Cadmus is meowing and that Cadmus is meowing are different facts, and it is not clear exactly what the difference between them consists in. But that these facts are different is presupposed by what Schaffer says. For instance, in defense of (f) Schaffer says 'the fact that the creature was produced from the meeting of this sperm and that ovum helps make it be Cadmus meowing, but doesn't help make it be Cadmus meowing' (2012: 129). And for my purposes here I do not need to clarify the difference between these facts. For, whatever the difference between these facts is, what Schaffer says suggests that the two facts are different. Furthermore, for (f) to be plausible, the grounded in (d) must be the fact that Cadmus is meowing, while the grounding in (e) must be the fact that Cadmus is meowing. And if this is so, then the present case is not a counterexample to the transitivity of grounding.

Thus Schaffer's case does not establish that grounding is not transitive. 
4. In this section I shall argue that truthmaking is not transitive. Consider (1) and (2):

(1) The fact that Socrates is white makes $<$ Socrates is white $>$ true.

(2) $<$ Socrates is white $>$ makes $<$ There are propositions $>$ true.

(1) is plausible for truthmaker theorists. And so is (2). For it is plausible that the truthmakers for an existential generalization are its instances if those instances are necessarily its instances. But it is also plausible that propositions are necessarily propositions. Thus any proposition is a truthmaker for $<$ There are propositions>. Now, transitivity would give us (3):

(3) The fact that Socrates is white makes $<$ There are propositions $>$ true.

But (3) is false. The proposition <There are propositions $>$ is not true in virtue of the fact that Socrates is white. Although the fact that Socrates is white makes true a truthmaker of <There are propositions $>$, the fact that Socrates is white is not that in virtue of which <There are propositions $>$ is true. Indeed, the fact that Socrates is white is not even that in virtue of which < Socrates is white> exists, and so one should not expect it to be that in virtue of which <There are propositions $>$ is true. Only that in virtue of which propositions exist can be the truthmaker of <There are propositions $>$. Thus (3) is false, and transitivity fails. ${ }^{6}$

Raven says that truthmaking cannot be a species of grounding because the relata of truthmaking are of different types and this precludes chaining (Raven 2013: 194; cf. Fine 2012: 44-45). But not all the relata of a truthmaking relation are of different types, as this example illustrates. A proposition can both be made true and make true another proposition. This is the case with $<$ Socrates is white > in the present example.

\footnotetext{
${ }^{6}$ After writing and submitting this paper I learnt, through a referee, of Aaron Griffith's paper, where he presents a counterexample to the transitivity of truthmaking very similar to mine (Griffith 2014: 205). He also denies the irreflexivity and asymmetry of truthmaking, though his examples for this claim are different from mine. More importantly, he seems to think that grounding is irreflexive, asymmetric and transitive (2014: 205).
} 
Is the alethic-fact relation non-transitive? I am not in a position to argue for such a claim. Consider, for instance, the following three claims:

(4) The fact that Socrates is white grounds the fact that $<$ Socrates is white $>$ is true.

(5) The fact that $<$ Socrates is white $>$ is true grounds the fact that $<$ There are propositions> is true.

(6) It is not the case that the fact that Socrates is white grounds the fact that $<$ There are propositions $>$ is true.

These three claims do not show that alethic-fact grounding is non-transitive, for although (4) and (6) are true, (5) is false. What <There are propositions $>$ is true in virtue of is not the fact that $<$ Socrates is white $>$ is true, but rather the fact that $<$ Socrates is white $>$ exists.

One might then think that the following three claims show that alethicfact grounding is non-transitive:

(4) The fact that Socrates is white grounds the fact that <Socrates is white $>$ is true.

(7) The fact that $<$ Socrates is white $>$ is true grounds the fact that $<$ There are true propositions $>$ is true.

(8) It is not the case that the fact that Socrates is white grounds the fact that $<$ There are true propositions $>$ is true.

But these three claims do not show that alethic-fact grounding is non-transitive. For although both (4) and (7) are true, I see no reason to accept (8) that does not assume that alethic-fact grounding is non-transitive. ${ }^{7}$

\footnotetext{
7 Points made by Nick Jones and Ezequiel Zerbudis made me see that I am not in a position to argue that alethic-fact grounding is non-transitive on the basis of the example based on claims
} 
But it is clear that (1)-(3) show that truthmaking is not transitive, and so truthmaking is not a strict order. And this is enough to show that grounding is not transitive, and so not a strict order either. But there are other reasons why truthmaking is not a strict order: it is neither irreflexive nor asymmetric, as we shall now see. Thus grounding is also neither irreflexive nor asymmetric, as we shall now see.

5. In this section I shall argue that grounding is not irreflexive. Consider the proposition A: <A exists $>$. This proposition says of itself that it exists. Let us suppose that A exists. If so, A is true. What makes it true? According to standard truthmaking ideas, a proposition of the form $<e$ exists $>$ is made true by $e$ itself. Thus, if it exists, A is made true by itself. Thus truthmaking is not irreflexive, and therefore neither is grounding.

But there is another reason why grounding is not irreflexive, since alethicfact grounding also fails irreflexivity. To see this, consider the truth-teller, $\mathrm{B}:<\mathrm{B}$ is true>. This proposition says of itself that it is true. Let us suppose that $B$ is indeed true. What grounds the fact that it is true? The fact stated by B itself. But what B states is that it itself is true. Thus, if B is true, the fact that B is true grounds the fact that $B$ is true. Therefore, alethic-fact grounding is not irreflexive.

(4), (7), and (8). Tuomas Tahko (2013) argues that what he calls truth-grounding is not transitive, and what he calls truth-grounding seems to be what I am calling here alethic-fact grounding. Simplifying a little bit, he argues that while the fact that bottle $b$ exists is grounded in the fact that $b$ has a stable macrophysical structure, and the fact that $b$ has a stable macrophysical structure is grounded in the fact that the Pauli Exclusion Principle holds, it is not the case that the fact that $b$ exists is grounded in the fact that the Pauli Exclusion principle holds (2013: 336-337). The problem with this is that it does not seem to counterexemplify the transitivity of truthgrounding in particular, since no fact involved in this case is a fact about something's being true. But I do not think the example even works against the transitivity of grounding in general. Tahko thinks that general laws like the Pauli Exclusion Principle in no way contribute towards answering the question about the existence of bottle $b$ (2013: 337). But if the fact that the Pauli Exclusion principle holds grounds the fact that $b$ has a stable macrophysical structure, and this latter fact grounds the fact that $b$ exists, then I see no reason, other than assuming that transitivity must fail, why the fact that the Pauli Exclusion Principle holds should not ground the fact that bottle $b$ exists or even the fact that <bottle $b$ exists $>$ is true. One might try reformulating the example as follows: the fact that $<b$ has a stable macrophysical structure $>$ is true grounds the fact that $<b$ exists $>$ is true, the fact that the Pauli Exclusion Principle holds grounds the fact that $<b$ has a stable macrophysical structure $>$ is true, but it is not the case that the fact that the Pauli Exclusion Principle holds grounds the fact that $<b$ exists $>$ is true. But this reformulation has the additional problem that the first claim, that the fact that $<b$ has a stable macrophysical structure $>$ is true grounds the fact that $<b$ exists $>$ is true, seems incorrect: what grounds the truth of $<b$ exists $>$ is, in any case, something about $b$, say that it has a stable macrophysical structure, but not something about the truth of another proposition. 
And since alethic-fact grounding is a species of grounding, grounding is not irreflexive. ${ }^{8}$ (Note that if $A$ is its own truthmaker, it is its only truthmaker, and if the fact that $B$ is true is grounded by the fact that $B$ is true, the fact that $B$ is true is its sole ground. This means that A is its own full ground and the fact that B is true is its own full ground). ${ }^{9}$

Kripke has proposed a theory of truth in which the concept of groundedness plays a central role. The truthbearers in Kripke's theory are sentences. On Kripke's theory, the truth-teller, the sentence that says of itself that it is true, is ungrounded (Kripke: 2011: 85, 91). Thus what I am proposing here seems to conflict with Kripke's theory of truth.

But it is important to appreciate the real point of conflict. On Kripke's theory that a sentence is ungrounded basically means that its truth-value is not ultimately determined by non-semantic facts (Kripke 2011: 88). The motivation of Kripke's theory is to give an explanation of truth that avoids the semantic paradoxes, and he does this in a way that accounts for how someone who initially does not understand the truth predicate, but who understands other non-semantic predicates, would learn to use the truth predicate (2011: 85). At the base of Kripke's construction there are sentences featuring only nonsemantic predicates. The assignment of truth-values to sentences featuring nonsemantic predicates determines a minimal fixed point in which the truth value of sentences featuring semantic predicates is ultimately determined by the truth value of sentences featuring only non-semantic predicates. The sentences that do not have a truth-value in the minimal fixed points are ungrounded. Thus for Kripke 'ungrounded sentence' means 'sentence not ultimately grounded in nonsemantic facts'.

But, as Kripke is of course aware, the truth-teller is not a paradoxical sentence. The truth-teller can consistently be assigned the truth-value true, and

\footnotetext{
8 Fine (2010: 104-105) says that there might be special cases in which something, in one capacity, is a ground for itself in another capacity, and that the truth-teller might in this sense be a ground for itself. Unfortunately he does not explain how the truth teller, in one capacity, can ground itself in another capacity. But, whatever grounding in one capacity might mean, in my example the truth-teller grounds itself in one and the same capacity.

${ }^{9}$ It is interesting to note that my counterexamples to the irreflexivity of grounding do not depend on its transitivity, which I have anyway rejected in the case of truthmaking. This distinguishes my counterexamples from Correia's, which assume the transitivity of grounding (Correia 2014: 54$55)$.
} 
Kripke's theory allows for this - the truth-teller has a truth-value in every maximal fixed point (2011: 91-92). But since in those cases the truth of the truth-teller is not determined by non-semantic facts at all, the truth-teller counts as a truth but an ungrounded truth (2011: 91-92).

Translated into my schema of propositions and facts about their being true, this becomes the idea that a proposition is ungrounded only if the fact that it is true is not ultimately determined by non-semantic facts. Now, in so far as Kripke would maintain that the fact that B is true is not determined by nonsemantic facts at all, I am not in conflict with him, since I maintain that the fact that $\mathrm{B}$ is true is not determined by non-semantic facts at all. But in so far as Kripke seems to maintain that the semantic can only be grounded in the nonsemantic, I disagree. I can see no reason why semantic facts, e.g. facts that a certain proposition is true, must be ultimately grounded in non-semantic facts. And, indeed, the example of proposition B convinces me that there are semantic facts ultimately grounded in semantic facts. Furthermore, the idea that the semantic can only be grounded in the non-semantic does not seem to be essential to Kripke's solution of the paradoxes. What is essential for that is simply that the truth value of certain sentences is not determined by nonsemantic facts at all. And this is independent of the idea that the semantic can only be grounded in the non-semantic.

Note, by the way, that my case that proposition A is made true by itself does not presuppose that semantic facts can be ultimate grounds. For proposition $\mathrm{A}$ is a proposition, not a fact, and therefore not a semantic fact. Nor is the fact that proposition A exists a semantic fact, since a semantic fact is a fact about whether a certain proposition is true or not. So even those who believe that semantic facts cannot be ultimate grounds will have reason to reject the irreflexivity of truthmaking and therefore that of grounding too.

Now, proposition B can consistently be thought of as true, it can consistently be thought of as false, and it can consistently be thought of as indeterminate; and there is no evidence about which one it is. So, it might be objected, there is no evidence that proposition $B$ is true, and therefore no evidence has been presented that grounding is not irreflexive. But this objection wrongly assumes that the relevant target is the thesis that there are no actual 
instances of reflexive grounding, i.e. no actual instances of entities grounding themselves. But this is not the thesis I am trying to refute. The thesis I am trying to refute is that it is conceptually necessary, or at least metaphysically necessary, that grounding is irreflexive. That is what those who maintain the irreflexivity of grounding maintain. And, in any case, that it is conceptually necessary, or at least metaphysically necessary, that grounding is irreflexive is what defenders of the irreflexivity of grounding should maintain. For when postulating that grounding is irreflexive (or asymmetric, or transitive) they are concerned with the most general and abstract formal features of grounding, not with aspects that might but need not be true of it. But if, under the non-contradictory assumption that $\mathrm{B}$ is true, it is shown that the fact that B is true would ground itself, then it is not conceptually true that alethic-fact grounding is irreflexive. And therefore the thesis that grounding is irreflexive, in the intended sense, has been refuted.

A similar point can be made about proposition A. For given that under the non-contradictory assumption that A exists, I have shown that A would be its own truthmaker, and so its own ground, I have shown that it is not conceptually true that grounding is irreflexive.

It might be claimed that although it is not conceptually necessary that alethic-fact grounding and truthmaking are irreflexive, it is metaphysically necessary that this is so. One way for this to happen is for it to be metaphysically necessary that if A exists, it is not its own truthmaker; or for it to be metaphysically necessary that if B is true, the fact that it is true is not grounded in itself.

Now, given that propositions of the form $<e$ exists $>$ are made true by $e$ itself, it is very difficult to see how A could exist without being made true by itself. Similarly, given that propositions are grounded in the facts stated by them, it is very difficult to see how the fact that B is true could fail to be grounded in itself.

So perhaps it is necessary that A does not exist? But why should this be the case? There is certainly no contradiction in its existence. And there are plenty of other self-referring objects. Indeed, there are some objects very similar to A. Consider this token sentence S: S does not exist. S does exist - it is located on this page. So why couldn't proposition A exist? What does not exist is the case for the 
necessary non-existence of A.

Depending on one's metaphysics of propositions, one might have doubts about the existence of propositions like A. For instance, if propositions are broadly Russellian in the sense of being complex objects containing the objects they are about, proposition A would contain itself as a proper constituent. This need not be a reason for rejecting self-referential propositions though, since the notion of something being a proper constituent of itself need not be absurd. But even if it is, there are other conceptions of propositions in which proposition $\mathrm{A}$ would not be a proper constituent of itself. Furthermore, the truthmaker theorist is unlikely to adopt any Russellian view of propositions, since these are too similar to facts, and he needs to maintain a clear distinction between facts and propositions.

How about the fact that B is true? Perhaps it is necessary that B is untrue? It may be argued that this is so because it is metaphysically necessary that truths are ultimately determined by non-semantic facts. Since the truth of B cannot be determined by non-semantic facts, then B cannot be true, and so the fact that B is true cannot exist.

But there is a problem with this line of thought, namely that if it is correct, there must be truths that are not ultimately grounded in non-semantic facts. For suppose that $B$ is untrue because it is not ultimately grounded in non-semantic facts. Consider then the proposition $\mathrm{B}^{*}$ : $<\mathrm{B}$ is untrue $>$. $\mathrm{B}^{*}$ is true, since, we are assuming, $\mathrm{B}$ is untrue. But the truth of $\mathrm{B}^{*}$ is not ultimately determined by nonsemantic facts: it is, on the contrary, ultimately determined by the semantic fact that $B$ is untrue.

Thus, there is not much prospect in arguing that although it is not conceptually necessary that grounding is irreflexive, it is metaphysically necessary that this is so.

It is interesting to note that my counterexamples to the irreflexivity of grounding and truthmaking share an important feature that not every other potential counterexample shares. The potential counterexample to the irreflexivity of grounding Jenkins considers is that of a pain being grounded in itself, by virtue of its being grounded in a brain state to which it is identical (Jenkins 2011: 271). This depends on a controversial metaphysics of mind. But 
there is another reason why this example is controversial. The idea that mental states are grounded in brain states is motivated by an interest in relating two distinct sets of states. It is difficult to see why an identity theorist should feel inclined to maintain that mental states are grounded in brain states. But truthmaker theory is committed to the claim that a proposition of the form $<e$ exists $>$ is made true by $e$, and so it entails that if proposition A exists, it is made true by itself. Similarly, the theory of grounding maintains that a fact that a certain proposition is true is grounded in the fact stated by that proposition, and so it entails that if proposition B is true, the fact that it is true is grounded in itself.

6. If truthmaking and alethic-fact grounding are asymmetric, they must be irreflexive. Their non-irreflexivity, however, guarantees their non-asymmetry only by guaranteeing that cases of symmetric truthmaking and alethic-fact grounding are cases of reflexive truthmaking and alethic-fact grounding. But if what I have said above is accepted, it will not be difficult to see that truthmaking and grounding are non-asymmetric in the full sense of there being distinct entities that ground each other.

Consider the propositions $\mathrm{C}=<\mathrm{D}$ exists $>$ and $\mathrm{D}=<\mathrm{C}$ exists $>$, and assume that both of them exist. The truthmaker of $C$ is $D$, and the truthmaker of $D$ is $C$. Therefore truthmaking is not asymmetric, and so neither is grounding.

Now consider the propositions $\mathrm{E}=<\mathrm{F}$ is true $>$ and $\mathrm{F}=\langle\mathrm{E}$ is true $>$, and assume they are both true. If so, given what they say, the fact that E is true grounds the fact that $\mathrm{F}$ is true, and the fact that $\mathrm{F}$ is true grounds the fact that $\mathrm{E}$ is true. ${ }^{10}$ Therefore, alethic-fact grounding is not asymmetric, and so neither is grounding.

But there is a natural objection to this. This is that grounding must be asymmetric because the ground is more fundamental than the grounded, and being more fundamental than is an asymmetric relation (cf. Rosen 2010: 116, Barnes 2014, Bennett forthcoming). The same kind of consideration can be used to argue that grounding must be irreflexive, since being more fundamental than is an irreflexive relation.

10 Thompson (2014) has given a similar example. 
Now, the relations of grounding and being more fundamental than are not the same relation. For something might be more fundamental than another thing without grounding it. This will be the case, for instance, if the former thing is fundamental and the latter one is grounded, but grounded in something other than the former thing. But the point that grounding must be irreflexive and asymmetric need not rely on an identification of the relations of grounding and being more fundamental than but only on the fact that when $x$ grounds $y, x$ must be more fundamental than $y$. But I shall argue that either the fact that $x$ grounds $y$ does not entail that $x$ is more fundamental than $y$ or there is no reason to think that it does.

Let us assume that the more fundamental than relation is asymmetric and irreflexive. But what is it to be more fundamental than something else? One answer is that it is to be closer to a fundamental (that is, ungrounded) entity than another thing (where it is understood that a fundamental entity is as close to a fundamental entity - itself - as anything can be). And the obvious way to understand this idea is that $x$ is closer to a fundamental entity than $y$ if and only if, in their chain of grounding, there are fewer elements between $x$ and a fundamental entity than between $y$ and a fundamental entity.

Now the relation of grounding is not restricted to finite chains. Nothing in the idea of grounding excludes the possibility of chains of grounding containing infinitely many distinct elements and such that there is no first element, no ungrounded ground. For instance, if wholes are grounded in their parts, a gunky whole, i.e. a whole all of whose parts have proper parts, would be at the top of an infinitely long chain of grounding with no first element. Now take two members of such a chain, $x$ and $y$, such that $x$ grounds $y$. Is $x$ more fundamental than $y$ ? No, for there is no fundamental entity in their chain of grounding, and therefore it is not the case that there are fewer elements between $x$ and a fundamental entity than between $y$ and a fundamental entity.

If so, that $x$ grounds $y$ does not entail that $x$ is more fundamental than $y$, and so the fact that reflexive and symmetric cases of grounding are cases in which the more fundamental than relation does not obtain between the ground and the grounded is no objection to them. Reflexive and symmetric cases of grounding are like bottomless chains of grounding: cases of grounding without 
different degrees of fundamentality. And in all three cases the reason why there are no different degrees of fundamentality is that there are no fundamental entities in the chains of grounding: neither bottomless chains of grounding, nor 'reflexive' chains of grounding, nor 'symmetric' chains of grounding, contain fundamental, ungrounded entities. ${ }^{11}$

It might be objected that there cannot be bottomless grounding chains, that is, that every chain must end in an ungrounded ground. I do not see why there cannot be such 'bottomless' chains. But I do not need their possibility to make my point. For it is sufficient that there can be dense chains of grounding, that is, chains of grounding such that between any two elements there is a further element. In this case it will also be true that no element of such a chain will be closer to a fundamental entity than any other element of it (except, of course, when the member in question is the ungrounded ground of the chain).

It might be objected that the real number line is dense but one can make sense of one positive real being closer to zero than another positive real. But this is because we can independently make sense of the notion of one positive real being bigger or smaller than another one. In this case we are working with a notion of relative fundamentality according to which $x$ is closer to a fundamental entity than $y$ if and only if, in their respective chains of grounding, there are fewer elements between $x$ and a fundamental entity than between $y$ and a fundamental entity.

A better objection would be against the characterization I have given of what it is to be more fundamental than something else. Perhaps $x$ is more

\footnotetext{
11 Note that there is a sense in which a self-grounder is more fundamental than something grounded in something else, even if the self-grounder is not an ungrounded entity. This has two consequences. One is that the idea of being more fundamental than, understood as being closer to a fundamental, ungrounded, entity, cannot be straightforwardly extended to apply to cases of entities belonging to different chains of grounding. For something that is its own sole ground is no closer to an ungrounded entity than something that is grounded in an ungrounded entity, and yet the former seems more fundamental than the latter. The other consequence is that a selfgrounder that grounds something else is more fundamental than what it grounds, even if it is not closer to an ungrounded entity. Thanks to Paul Audi for making me think of these points. The solution is to redefine, in the context of the present definition of being more fundamental than, a fundamental entity as one that is either ungrounded or has no grounds other than itself. Even under this definition of a fundamental entity it is still the case that no member of an infinitely long descending chain of grounding will be closer to a fundamental entity than any other member of it, and that the members of 'reflexive' and 'symmetric' chains of grounding (at least the kinds of such chains I have considered in this paper) will not be closer to a fundamental entity than any other member of them.
} 
fundamental than $y$ if and only if every ground of $x$ is a ground of $y$ but not vice versa? This definition is satisfied by suitable pairs of elements in grounding chains with infinitely many elements, whether such chains have an ungrounded first element or not. ${ }^{12}$ But, if this is what being more fundamental than is, what reasons are there for thinking that if $x$ grounds $y, x$ must be more fundamental than $y$ ? There do not seem to be any. Indeed, there seem to be no reasons why if $x$ grounds $y$ then everything in virtue of which $x$ is F is something in virtue of which $y$ is $\mathrm{F}$, but not vice versa. Furthermore, my counterexamples to the irreflexivity and the asymmetry of grounding are counterexamples to the idea that if $x$ grounds $y$, every ground of $x$ must be a ground of $y$ but not vice versa. So, not only do there seem to be no reasons to think that if $x$ grounds $y, x$ must be more fundamental than $y$ in this sense, there are actually reasons against it.

Finally, one might say that $x$ 's being more fundamental than $y$ means that $x$ grounds $y$ but is not grounded in it. But, again, there seem to be no reason for thinking that if this is what being more fundamental than something means, then grounding something entails being more fundamental than it. For there is no reason to think that if $x$ grounds $y$ then, although $y$ is $\mathrm{F}$ in virtue of $x, x$ is not $\mathrm{F}$ in virtue of $y$. And, again, my examples constitute reasons against the idea that if $x$ grounds $y$ then $x$ is not grounded in $y$.

Analogous replies could be made if someone objected that truthmaking must be asymmetric because if $x$ is a truthmaker of $y, x$ must be more fundamental than $y$.

So these objections can be repelled. If my point that grounding is not asymmetric stands, it follows that grounding does not entail being more

\footnotetext{
12 Three comments on the definition. (a) According to this definition a fundamental thing is more fundamental than what it grounds since they vacuously satisfy the condition that every ground of the fundamental thing is a ground of what it grounds and non-vacuously satisfy the condition that not every ground of what the fundamental thing grounds is a ground of it. (b) As formulated, this definition restricts the relation of being more fundamental than to pairs of things that are elements of the same grounding chain. If something like this definition is correct, then whether it can be extended to apply to pairs of things from different chains should be investigated. But this is not the topic of this paper and the objection I am considering does not depend on whether or not such a definition can be thus extended. (c) As formulated, this definition assumes the transitivity of grounding. A more careful formulation of the definition, which does not assume the transitivity of grounding, says that $x$ is more fundamental than $y$ if and only if the transitive closure of the grounds of $x$ is a subset of the transitive closure of the grounds of $y$, but not vice versa. But it is only for simplicity that I gave the less careful formulation and, anyway, the point I make in the text against the less careful version of the definition would also apply to the more careful version.
} 
fundamental than. Thus it follows that grounding is not a relation of relative fundamentality, to use an expression of Bennett's (Bennett, forthcoming).

But there is another possible objection to my arguments that grounding is not asymmetric. This is that grounding is a kind of explanation and explanations are asymmetric. For instance, Fine says that 'there is a plausible demand on ground or explanation that we are unable to evade. For given a truth that stands in need of explanation, one naturally supposes that it should have a "completely satisfactory" explanation, one that does not involve cycles and terminates in truths that do not stand in need of an explanation' (Fine 2010: 105). This appeal to the asymmetry of explanation to defend the asymmetry of grounding is not uncommon (Audi 2012a: 102, Rosen 2010: 116).

Now, it is true that explanations, in the sense of propositions put forward by an enquirer to advance our understanding, are asymmetric. Let us call explanations in this sense epistemic explanations. But there is no reason to extrapolate the formal features of epistemic explanations to grounding. I think that there is a close connection between epistemic explanations and grounding, namely that explanations track determinative relations and since grounding is a determinative relation, many explanations track grounding relations (cf. Rodriguez-Pereyra 2005: 28). But it does not follow that grounding must be asymmetric because epistemic explanations are. Indeed, it does not even follow that every relation of grounding must be susceptible of being tracked or represented by an epistemic explanation.

But the thought might be that there is a non-epistemic asymmetric sort of explanation and that grounding is such a non-epistemic sort of explanation or is at least a species of it. ${ }^{13}$ Something like this is what Fine seems to be assuming in the passage I quoted above, when he speaks of 'grounding or explanation'. But, whatever Fine was assuming, the idea that grounding is a non-epistemic asymmetric sort of explanation cannot be used as an objection against my points

\footnotetext{
${ }^{13}$ Here non-epistemic explanation should not be equated with metaphysical explanation in the sense in which the latter is contrasted with causal explanation. Both metaphysical and causal explanations can be types of epistemic explanations - the difference is that one tries to illuminate a phenomenon by pointing to its causal history while the other tries to illuminate a phenomenon by pointing to some kind of metaphysical antecedent. If equated with metaphysical explanation, non-epistemic explanation should be equated with it in the sense that the latter is contrasted with epistemic explanation.
} 
that grounding is not asymmetric. For in that case the objector would be saying: your thesis that grounding is not asymmetric must be wrong because grounding is asymmetric. Such objection has no force.

But I have argued that grounding is not asymmetric. It follows that grounding is not a non-epistemic asymmetric explanatory relation.

7. Schaffer has proposed a contrastive account of grounding according to which grounding is a quaternary relation of the form: the fact that $\mathrm{F}$ rather than the fact that $\mathrm{F}^{*}$ grounds the fact that $\mathrm{G}$ rather than the fact that $\mathrm{G}^{*}$, where the facts that $\mathrm{F}$ and $\mathrm{G}$ are required to be obtaining facts and the facts that $\mathrm{F}^{*}$ and $\mathrm{G}^{*}$ are required to be non-obtaining facts. Schaffer thinks that grounding respects the principles of transitivity, asymmetry, and reflexivity when these are formulated for differences. Thus:

Differential irreflexivity: It is not the case that the fact that $\mathrm{F}$ rather than $\mathrm{F}^{*}$ grounds the fact that $\mathrm{F}$ rather than $\mathrm{F}^{*}$.

Differential asymmetry: If the fact that $\mathrm{F}$ rather than $\mathrm{F}^{*}$ grounds the fact that $\mathrm{G}$ rather than $\mathrm{G}^{*}$, then it is not the case that the fact that $\mathrm{G}$ rather than $\mathrm{G}^{*}$ grounds the fact that $\mathrm{F}$ rather than $\mathrm{F}^{*}$

Differential transitivity: If the fact that $\mathrm{F}$ rather than $\mathrm{F}^{*}$ grounds the fact that $\mathrm{G}$ rather than $G^{*}$, and the fact that $G$ rather than $G^{*}$ grounds the fact that $H$ rather than $\mathrm{H}^{*}$, then the fact that $\mathrm{F}$ rather than $\mathrm{F}^{*}$ grounds the fact that $\mathrm{H}$ rather than $\mathrm{H}^{*}$.

Schaffer argues that his counterexamples to the transitivity of grounding are not counterexamples to the differential transitivity of grounding. And he suggests (2012: 133, fn. 17) that Jenkins' counterexample to the irreflexivity of grounding does not threaten differential irreflexivity. This suggests that a quaternary relation of grounding may be a strict order.

But my examples are also examples against differential irreflexivity and differential asymmetry of grounding. The fact that $B$ is true rather than $B$ is not true grounds the fact that B is true rather than B is not true. So differential 
irreflexivity fails. And the fact that $\mathrm{E}$ is true rather than $\mathrm{E}$ is not true grounds the fact that $\mathrm{F}$ is true rather than $\mathrm{F}$ is not true, and the fact that $\mathrm{F}$ is true rather than $\mathrm{F}$ is not true grounds the fact that $\mathrm{E}$ is true rather than $\mathrm{E}$ is not true. So differential asymmetry fails.

Therefore, taking grounding to be a quaternary relation does not make it a strict order.

8. To conclude, truthmaking is neither transitive, nor irreflexive, nor asymmetric, and alethic-fact grounding is neither irreflexive nor asymmetric. Since those two relations are species or cases of grounding, grounding is neither transitive, nor irreflexive, nor asymmetric, and so grounding is not a strict order. This has some consequences. One consequence of this is that if $x$ grounds $y$, it does not follow that $x$ is more fundamental than $y$. Thus grounding is not a relation of relative fundamentality. Another consequence of this is that, if non-epistemic explanation is an asymmetric relation, grounding is not non-epistemic explanation. The link between grounding and explanation consists in that instances in which grounding occurs irreflexively and asymmetrically back up epistemic explanations.

\section{References}

Armstrong, D. M. 2004. Truth and Truthmakers. Cambridge: Cambridge University press.

Audi, P. 2012a. 'A clarification and defense of the notion of grounding', in F. Correia and B. Schnieder (eds.), Metaphysical Grounding: Understanding the Structure of Reality. Cambridge: Cambridge University Press, pp. 101-121.

Audi, P. 2012b. 'Grounding: toward a theory of the in virtue of relation', The Journal of Philosophy, CIX/12, pp. 685-711. 
Barnes, E. 2014. 'Symmetric dependence', unpublished MS, http://www.personal.leeds.ac.uk/ phlejb/symmetric\%20dependence.pdf

Bennett, K. Making things up, forthcoming in Oxford University Press.

Correia, F. 2010. 'Grounding and truth-functions', Logique et Analyse 53/211, pp. 251-279.

Correia, F. 2014. 'Logical grounds', The review of Symbolic Logic, 7/1, pp. 31-59.

Fine, K. 2010. 'Some puzzles of ground', Notre Dame Journal of Formal Logic, 51/1, pp. 97-118.

Fine, K. 2012. 'Guide to ground', in F. Correia and B. Schnieder (eds.), Metaphysical Grounding: Understanding the Structure of Reality. Cambridge: Cambridge University Press, pp. 37-80.

Griffith, A. 2014. 'Truthmaking and grounding', Inquiry, 57/2, pp. 196-215.

Jenkins, C. 2011. 'Is metaphysical dependence irreflexive?', The Monist, 94/2, pp. 267-276.

Kripke, S. 2011. 'Outline of a theory of truth', in S. Kripke, Philosophical Troubles, New York: Oxford University Press, pp. 75-98.

Liggins, D. 2012. 'Truth-makers and dependence', in F. Correia and B. Schnieder (eds.), Metaphysical Grounding: Understanding the Structure of Reality. Cambridge: Cambridge University Press, pp. 254-271.

Litland, J. E. 2013. 'On some counterexamples to the transitivity of grounding', Essays in Philosophy, 14/1, http://dx.doi.org/10.7710/1526-0569.1453 
Raven, M. 2013. 'Is ground a strict partial order?' American Philosophical Quarterly, 50/2, pp. 193-201.

Rodriguez-Pereyra, G. 2002. Resemblance Nominalism: a Solution to the Problem of Universals. Oxford: Oxford University Press.

Rodriguez-Pereyra, G. 2005. 'Why truthmakers', in J. Dodd and H. Beebee (eds.), Truthmakers: the contemporary debate. Oxford: Oxford University Press, pp. 1731.

Rosen, G. 2010. 'Metaphysical dependence: grounding and reduction', in B. Hale and A. Hoffman (eds.) Modality: Metaphysics, Logic, and Epistemology. New York: Oxford University Press, pp. $109-36$.

Schaffer, J. 2009. 'On what grounds what', in D. Chalmers, D. Manley and R. Wasserman (eds.), Metametaphysics: new essays on the foundations of ontology. Oxford: Oxford University Press, pp. 347-383.

Schaffer, J. 2012. 'Grounding, transitivity, and contrastivity', in F. Correia and B. Schnieder (eds.), Metaphysical Grounding: Understanding the Structure of Reality. Cambridge: Cambridge University Press, pp. 122-138.

Schnieder, B. 'Truth-making without truth-makers', Synthese, 152/1, pp. 21-46.

Tahko, T. E. 2013. 'Truth-grounding and transitivity', Thought, 2/4, pp. 332-40.

Thompson, N. 2014. 'Metaphysical interdependence', unpublished MS. 